\title{
Correction to: New algorithms for fixed-length approximate string matching and approximate circular string matching under the Hamming distance
}

\author{
ThienLuan $\mathrm{Ho}^{1}$. Seung-Rohk $\mathrm{Oh}^{1}$. \\ HyunJin $\operatorname{Kim}^{1}$ (iD
}

Published online: 20 March 2018

(C) Springer Science+Business Media, LLC, part of Springer Nature 2018

\section{Correction to: J Supercomput https://doi.org/10.1007/s11227-017-2192-6}

The funding information is missing in the Acknowledgements section of the original article. The correct wording is given below.

Acknowledgements We would like to thank Mr. Ji-Won Song, MS. candidate at School of EEE in Dankook University, who have helped us set up the Linux-based experimental environment using GPU. This research was supported by Basic Science Research Program through the National Research Foundation of Korea (NRF) funded by the Ministry of Education (2017R1D1A1B03030348).

The original article can be found online at https://doi.org/10.1007/s11227-017-2192-6.

$\triangle$ HyunJin Kim hyunjin2.kim@gmail.com; hyunjin2@dankook.ac.kr

1 School of Electronics and Electrical Engineering, Dankook University, Yongin-si, Gyeonggi-do, Republic of Korea 